\title{
Recommendations for Analgesia and Sedation in Critically ill Children Admitted to Intensive Care Unit
}

Angela Amigoni ( $\square$ angela.amigoni@aopd.veneto.it )

University Hospital, Padova https://orcid.org/0000-0001-5654-0506

Giorgio Conti

Policlinico A Gemelli

Alessandra Conio

Regina Margherita Children's Hospital

Manuela Corno

ASST Papa Giovanni XXIII

Paola Claudia Fazio

Azienda Ospedale Universita Padova

Federica Ferrero

Novara Hospital

Marta Gentili

Ospedale Luigi Sacco

Cristina Giugni

AOU Meyer

Manuela L'Erario

AOU Meyer

Maria Maristella Masola

Azienda Ospedale Universita Padova

Paola Moliterni

Papa Giovanni XXIII Hospital

Giuseppe Pagano

University of Verona

Zaccaria Ricci

AOU Meyer

Stefano Romagnoli

Careggi University -Hospital

Beatrice Vasile

Azienda Ospedaliera Spedali Civili di Brescia

Francesca Vitale

Policlinico A Gemelli

Geremia Zito Marinosci 
Santobono Pausilipon Azienda Ospedaliera Pediatrica

Maria Cristina Mondardini

University Hospital IRCCS S.Orsola, Bologna

\section{Research Article}

Keywords: sedation, Pediatric Intensive Care Unit, pain, substance withdrawal syndrome, delirium

Posted Date: August 11th, 2021

DOl: https://doi.org/10.21203/rs.3.rs-774639/v1

License: (9) This work is licensed under a Creative Commons Attribution 4.0 International License. Read Full License

Version of Record: A version of this preprint was published at Journal of Anesthesia, Analgesia and Critical Care on February 12th, 2022. See the published version at https://doi.org/10.1186/s44158-02200036-9. 


\section{Abstract}

We aim to develop evidence-based recommendations for intensivists caring for children admitted to intensive care units and requiring analgesia and sedation.

A panel of national pediatric intensivists expert in the field of analgesia and sedation and other specialists (a pediatrician, a neuropsychiatrist, a psychologist, a neurologist, a pharmacologist, an anesthesiologist, two critical care nurses, a methodologist) started in 2018 a 2-years process. Three meetings and one electronic-based discussion were dedicated to the development of the recommendations (presentation of the project, selection of research questions, overview of text related to the research questions, discussion of recommendations). A telematic anonymous consultation was adopted to reach the final agreement on recommendations. A formal conflict-of-interest declaration was obtained from all the authors.

Eight areas of direct interest and one additional topic were considered to identify the best available evidence and to develop the recommendations using the Evidence-to-Decision framework according to Grading of Recommendations Assessment, Development and Evaluation (GRADE) approach. For each recommendation, the level of evidence, the strength of the recommendation, the benefits, the harms and the risks, the benefit/harm balance, the intentional vagueness, the values judgment, the exclusions, the difference of the opinions, the knowledge gaps and the research opportunities were reported.

The panel produced 17 recommendations. Nine were evaluated as strong, 3 as moderate, 5 as weak.

Conclusion: a panel of national experts achieved consensus regarding recommendations for the best care in terms of analgesia and sedation in critically ill children.

\section{What Is Known:}

- Monitoring the level of analgesia and sedation, symptoms of withdrawal syndrome and delirium is necessary to guarantee the best care of patients.

- latrogenic withdrawal syndrome is a critical complication of pediatric patients admitted to the intensive care unit that leads to recovery delays and worse outcomes.

- Acting on modifiable factors is a priority to prevent delirium $f$

What is new:

- As a first-line analgosedative therapy, opiates should be associated with an alfa agonist, decreasing the use of benzodiazepines due to their risk of increasing delirium

- In difficult analgosedation ketamine should be preferred to other molecules

- Good quality of sleep is a very important goal to be maintained in critically ill patients

\section{Introduction}


Analgesia and sedation are essential in the care of critically ill children. This is particularly true during intubation and mechanical ventilation, but controlling pain and agitation, reducing discomfort, allowing invasive procedures, avoiding accidental removal of medical devices are other crucial goals to achieve in these patients. Furthermore, pain control promotes the reduction of oxygen demand, and this result can only be achieved using a goal-directed strategy of treatment and a validated assessment of the patient's level of comfort. Under and over treatment are both harmful. Under-sedation doesn't allow to obtain adequate physiological and physical distress control. Conversely, over sedation may delay recovery, cause tolerance and possibly promote the development of withdrawal syndrome.

In 2006 Playfor et al [1], for the United Kingdom Pediatric Intensive Care Society Sedation Analgesia and Neuromuscular Blockade working Group, published consensus guidelines on analgesia and sedation in critically ill children and underlined the paucity of high-quality literature on this topic. This publication represents a milestone for this topic in pediatric age, especially because it gives clinicians the message to plan a targeted level of sedation for each patient and to regularly review it.

In 2014 an expert panel of the Italian Society of Neonatal and Pediatric Anesthesia and Intensive Care (SARNePI) developed 37 evidence-based recommendations. [2] Two years later, the Pediatric Cardiac Intensive Care Society produced a consensus statement dedicated to pharmacotherapy in cardiac critical care and focused to sedation, analgesia and muscle relaxant treatment. [3] In 2016 the European Society of Pediatric and Neonatal Intensive Care (ESPNIC) published a position statement to recommend accurate monitoring of pain and non-pain related distress in neonates and children admitted to Intensive Care Unit (ICU) and adoption of validate tools to evaluate level of sedation, iatrogenic withdrawal syndrome (WS) and delirium. [4]

However, nowadays a standardized approach to analgesia and sedation in Italy does not exist and single centres follow internal protocols, with high heterogeneity across the country. Through the development of national recommendations, the variations in practice may be reduced, thus improving patient's care.

The present document was produced through a consensus of national experts in intensive care. It covers many different perspectives, providing recommendations and research priorities.

These recommendations aim to assist clinicians and nurses in performing adequate analgesia and sedation to $0-18$ aged critically ill children (excluding neonates). The document is intended both for pediatric and adult intensivists who care for children admitted to intensive care units.

\section{Materials And Methods}

\section{Selection of the panel members}

In may 2018 the SARNePI appointed two co-chairs (coordinators of the SARNePI Pediatric Neurological Protection and Drugs study group) to carry out this project. The selection of panel members was conducted by the co-chairs, considering expertise in analgesia and sedation or in associated aspects. The 
panel was composed by 13 pediatric intensivists recruited from 12 Pediatric Intensive Care Units (PICUs), a pediatrician, a neuropsychiatrist, a psychologist, a neurologist, a pharmacologist, an anesthesiologist, two critical care nurses, a methodologist. Out of a group of 22 panellists, 16 (13 paediatric intensivists, 2 critical care nurses, a paediatrician) participated in the development of consensus on recommendations.

All panel members declared any potential conflicts. All of them had no conflict of interest to declare.

\section{Question development}

In 2018, following the National regulation for Guideline production, the panel convened a meeting to discuss the project and to identify the main clinical topics. Seven areas of interest (topics) were initially considered: analgesia and sedation, difficult analgesia and sedation, neuromuscular blocking, sleep, delirium, withdrawal syndrome, palliative sedation. During the second meeting, one additional topic was added: analgesia and sedation in children with developmental delay. Moreover, a section with the caregivers' opinion was included, assessed through a questionnaire to be administered in 4 PICUs. For each topic, the working group identified the research questions, according to the PICO format, which describes the population $(P)$, intervention $(I)$, control $(C)$ and outcomes $(0)$.

\section{Search strategy and evidence summation}

For each PICO a search strategy of literature was formulated. Search adopted a combination of controlled vocabulary (e.g. "pediatrics," "pediatric critical care", "intensive care unit") and keywords (e.g. "pain: acute, measurement, assessment, management", "sedation", "deep sedation", "conscious sedation", "iatrogenic withdrawal syndrome", "abstinence syndrome", "substance withdrawal syndrome”, "delirium"). Keywords were combined with "AND" pediatric critical care "OR" Intensive Care Unit.

PUBMED, SCOPUS, EMBASE, COCHRANE, CINHAIL databases were considered.

All studies were selected using the filter of age (birth to 18 years) and language (English). Studies including only neonates were excluded.

The search was applied to the following period: January 1 2008- December 31-2019. For some topics, papers older than 2008 were included due to the paucity of literature.

Panellists selected literature considering systemic reviews or meta-analysis, randomized controlled trials (RCT), observational studies, case series, case reports, narrative reviews were appropriated. Editorials and letters to the Editor were excluded. Studies published after the strategy search date were not included.

After the first selection, 243 papers were evaluated with a full text analysis and 165 relevant studies were included for the PICO development. (Fig. 1) Nine RCT and 51 non-randomised observational prospective studies were assessed to evaluate the risk of bias by panel members, working blinded, in couples. The Cochrane risk of bias tool was adopted for randomized trials [5] and the Newcastle-Ottawa Scale [6] for non-randomised prospective observational studies. The final agreement between the pairs was achieved through an electronic-based discussion. 


\section{Formulation of recommendations}

PICO development was discussed during the third meeting. Seventeen recommendations were formulated, 16 on the 8 topics and one on a special issue (communication) added to the text and related to caregivers' opinion. For each recommendation, were reported: the level of evidence, the strength of recommendation, the benefits, the harms and risks, the benefit-harm balance, the intentional vagueness (if applicable), the value judgment, the exclusions (if applicable), the difference of opinion between the panel members, the knowledge gaps and research opportunities.

To designate the levels of recommendations the panel followed the American Academy of Pediatrics policy statement "Classifying Recommendations for Clinical Practice" [7] with the application of the Evidence-to-Decision (EtD) framework according to Grading of Recommendations Assessment, Development and Evaluation (GRADE) approach.

Recommendations were developed by the two chairs considering published literature, following an evidence-based approach to provide evidence-based recommendations, considering risks and benefits and applicability. Recommendations should have been discussed during the fourth meeting but, due to the Corona Virus Disease - 2019 lockdown, the members used telematic communication to reach a final agreement. For each recommendation, a telematic anonymous agreement was adopted and $100 \%$ of consensus was reached. The strength of recommendations was finally classified as strong, moderate or weak. Strong recommendations started with "We recommend", moderate or weak recommendations started with "We suggest". Concerning the strength of recommendation, $75 \%$ of consensus was considered.

\section{Quality assessment}

These recommendations were developed considering the AGREE II (Appraisal Guideline REsearch Evaluation) Reporting Checklist. [8]

The draft version of this document underwent peer review and approval by representatives of the following scientific society: SARNePI, Italian Society of Analgesia Anesthesia and Intensive Care (SIAARTI), Medical and Nursing Academy of Paediatric Emergency and Intensive Care (AMIETIP), National Association of Critical Care Nurse (ANIARTI). Comments were reviewed by the co-chairs and incorporated into the recommendations as appropriate.

We planned to revise the recommendations every 5 years with the possibility to update them earlier if relevant literature on the topics will be published.

\section{Dissemination and implementation}

Dissemination of these recommendations will occur by publication in the websites of relevant scientific societies, national and international journals, presentations at national and international conferences, 
education sessions and meetings with staff at the individual institution level to assess the need for local adaptation.

To assess the impact of the recommendations on the care of patients, we design to launch a national survey one year after the publication of the document, particularly analysing the following indicators: incidence of withdrawal syndrome, incidence of delirium, rate of monitoring of the level of sedation, withdrawal syndrome, delirium, adopting respectively the Comfort Behaviour Scale (CBS), the Withdrawal Assessment Tool - 1, (WAT-1) and the Cornell Assessment Pediatric Delirium (CAPD).

\section{Summary of recommendations}

1. As a first-line strategy, we suggest optimizing analgesia using opiates and adopting alpha agonists as sedative agents, considering benzodiazepines a second-line.

2. We suggest adopting protocols of analgesia and sedation to administer the minimal effective dose of analgesics and sedatives to reduce tolerance and the incidence of difficult analgesia/sedation. Furthermore, the daily interruption of sedation should be considered with caution.

3. We recommend regular monitoring with validated tools the level of analgosedation of pediatric patients admitted to ICU.

4. In difficult analgesia/sedation we suggest using ketamine, due to its good safety profile.

5. We suggest using neuromuscular blocking agents in patients with severe respiratory insufficiency and persistent patient-ventilator asynchrony despite actions taken to limit the rate of asynchrony.

6. We suggest monitoring the level of sedation with continuous processed EEG in patients treated with neuromuscular blocking agents, considering the limitation and availability of the device.

7. We recommend adopting in all pediatric patients admitted to ICU strategies to prevent sleep alterations, particularly non-pharmacologic ones (relaxing techniques, parental involvement, control of environmental factors)

8. We recommend working on modifiable risk factors, particularly reducing the use of benzodiazepines.

9. We suggest basing the treatment of pediatric delirium on maximizing preventive bundles. Antipsychotic drugs may be used with careful consideration of contraindications.

10. We recommend regular monitoring delirium in critically ill children every day of the ICU stay, using validated tools.

11. We recommend working on modifiable risk factors of WS, particularly avoiding weaning higher than a daily reduction of $20 \%$ respect on the initial dose

12. We recommend treating WS with additional boluses of the drug considered to be responsible for the symptoms and modifying the weaning plan.

13. We recommend regular monitoring withdrawal symptoms in critically ill children treated with analgesics and/or sedatives longer than 72 hours, adopting validated tools.

14. We recommend performing pediatric palliative sedation (PPS) early defining an interdisciplinary plan agreed with parents.

15. We suggest adopting a personalized strategy to achieve PPS in children, to ensure the maximal efficacy using doses tailored to the patient 
16. In children with developmental delay, we suggest adopting validated tools to monitor the level of sedation, the presence of delirium and WS in ICU, considering their limitations and involving the caregivers.

17. We recommend explaining to parents the meaning of analgesia and sedation and off-label drugs. If analgesia and sedation lasted more than 48 hours, we recommend informing parents about the risk of WS and delirium development.

\section{Analgesia And Sedation}

Children admitted to Intensive Care Unit are exposed to pain due to clinical situations and procedures. An adequate level of sedation may be reached only if pain is absent. Acute pain needs to be immediately identified by physicians and nurses [4], otherwise it may become chronic. Chronic pain is difficult to treat and requires a specific approach. [9]

Clinicians need to plan an "ideal" goal of sedation for each patient. Generally, a state of a quiet, sleepy child requiring stimulus to being awake without distress and excessive movements is considered an adequate level of sedation during mechanical ventilation.

Adequate analgesia and sedation depend on pharmacologic and non-pharmacologic treatment, but also environmental factors. [10]

Over-sedation is correlated with increased length of ventilation and ICU stay, with increased incidence of WS and delirium. Under-sedation is correlated with patient's discomfort and adverse events related to the risk of devices removal.[11]

WHICH IS THE BEST PHARMACOLOGIC TREATMENT TO ENSURE AN ADEQUATE ANALGESIA AND SEDATION IN CHILDREN IN ICU?

Opiates are the most common analgesic drugs adopted in Pediatric Intensive care Unit (PICU), especially morphine and synthetic opiates (fentanyl, remifentanil, sufentanil). Remifentanil seems to promote a more rapid weaning from ventilation compared to fentanyl, but, due to its ultra-brief half-life, the risk of developing tolerance and hyperalgesia increases, particularly with high doses. [12] Some authors consider remifentanil the ideal opiate in ventilated infants, due to a low risk of accumulation. In a singlecentre, double-blind RCT the median extubation time was significantly shorter in the remifentanil group but results are strongly conditioned by the low sample size. [13]

Genetic polymorphism promotes the individual variability of drug response and the possibility to develop chronic pain. Facilitating and protective genotypes were identified, some of them correlated with the efficacy of analgesic drugs, others to the development of toxicity. A recent review reported 10 genotypes involved in individual response to opiates. [14] In particular, genotype ABCB1 seems to be involved. Children with allele AA are described to require a lower dose of fentanyl compared to AG or GG alleles. [15] Recent studies on morphine and midazolam in ventilated children reported a possible role of UGT2BT 
polymorphism in midazolam metabolism. [16,17] Therefore, genotype identification may be relevant to predict dosing requirements and treatment efficacy.

For perioperative pain management, in 2018 a clinical guidance (ESPA Pain management Ladder) was published by the European Society for Paediatric Anesthesiology. [18]

Considering sedatives, in PICU the most used drugs are benzodiazepines, particularly midazolam. Italian data were reported by Tabacco et al. [19]

However, concerns about benzodiazepines have recently emerged. Drugs acting on $\gamma$ - aminobutyric acid (GABA) receptor might promote a neurotoxic effect especially in patients younger than 3 years. [20,21] Moreover, a direct and dose-dependent association of benzodiazepine with the development of delirium in critically ill children has been reported. [22,23]

At present, barbiturates were used in status epilepticus, in procedural sedation [21] and pediatric brain trauma with refractory intracranial hypertension. [24]

In the last years, the use of alpha-agonists as adjuvants increased, also in Italy. [25] In 2015, the Italian Medicines Agency (AIFA) admitted the use of dexmedetomidine in critically ill children refractory to conventional treatment. [26] Wolf et al, in a double-blind RCT, considered efficacy and safety reporting a non-inferiority of clonidine versus midazolam. [27] Recently, interest turned to dexmedetomidine, a drug with more specific stimulation of alpha 2 receptors. A review published in 2016 reported a sparing effect of dexmedetomidine compared to opiates and benzodiazepine, with a good safety profile. [28] In the same year dexmedetomidine was evaluated in a large study on pediatric ventilated patients, analysing the use of this drug as first-line therapy, as second-line therapy and as a before-extubation strategy. In the group of patients treated with dexmedetomidine as first-line therapy a good level of sedation (measured with a validated scale) was reached. The use of dexmedetomidine before extubation was effective in reducing the length of ventilation. [29] In children submitted to cardiac surgical procedure this result was confirmed. [30] A retrospective large monocentric study reported a good efficacy of dexmedetomidine as a single sedative agent in non-invasive ventilated pediatric patients.[31]

\section{RECOMMENDATION 1:}

As a first-line strategy, we suggest optimizing analgesia using opiates and adopting alpha agonists as sedative agents, considering benzodiazepines a second-line.

\section{Strength of recommendation: Moderate}

\section{ARE PROTOCOLS OF ANALGOSEDATION USEFUL IN CHILDREN ADMITTED TO ICU?}

Data on adults reported the efficacy of protocols of analgesia and sedation in reducing complications of ICU stay. In the pediatric population their efficacy is less strong and only a few studies have proven a positive impact of nurse-driven protocols. In a multicentre cluster-randomized trial, the use of a sedation 
protocol did not reduce the duration of mechanical ventilation. Nevertheless, patients in the intervention group were exposed to fewer days of opioid exposure and sedative classes. [32] Some authors compared phase before and after protocol implementation, showing a reduction in analgesic and sedative doses and in tolerance development of tolerance.

Particularly, after protocol implementation were reported: a reduction in the duration of treatment with opiates and benzodiazepine [33], a reduction in the incidence of WS [34, 3, 35, 36] a reduction of daily doses of benzodiazepine and a reduction in the duration of mechanical ventilation in children older than 12 months [37], a reduction of cumulative doses of benzodiazepine and WS in surgical patients. [38] Nevertheless, the only implementation of a protocol itself doesn't improve the quality of care for a prolonged time, because reinforce strategies need to be maintained. [33, 39]

In adults admitted to ICU, daily interruption of sedation was reported to decrease the duration of mechanical ventilation and of ICU stay. Cumulative doses of benzodiazepines also decreased. In the pediatric population RCT are scarce and their results are questionable. The first RCT showed a reduction of the length of mechanical ventilation, PICU stay and cumulative dose of midazolam in patients treated with interrupted sedative infusion versus continuous infusion. [40] Conversely, a later RCT reported that daily interruption of sedation doesn't obtain these results but promotes more frequent periods of undersedation. [41] In a more recent RCT, daily sedation interruption was evaluated in addition to protocolized sedation, finding that daily sedation interruption did not reduce the duration of mechanical ventilation, ICU stays, or amounts of sedatives, and was associated with increased mortality. For this reason, the authors don't recommend this strategy of treatment. [42]

Nowadays, protocols based on drug rotation of analgesic and/or sedatives don't find the support of the literature.

\section{RECOMMENDATION 2:}

We suggest adopting protocols of analgesia and sedation to administer the minimal effective dose of analgesics and sedatives to reduce tolerance and the incidence of difficult analgesia/sedation. Furthermore, the daily interruption of sedation should be considered with caution.

\section{Strength of recommendation: Moderate}

WHICH IS THE ADEQUATE MONITORING OF ANALGESIA AND SEDATION IN PEDIATRIC PATIENTS ADMITTED TO ICU?

It is challenging to distinguish between pain, distress, WS and delirium in critically ill children because symptoms - depending on patient's age-are often not specific and may overlap. For this reason, it is of priority importance to include in every protocol of analgesia and sedation regular monitoring of patients with validated scales $[4,21]$ to evaluate the level of analgesia, sedation, the presence of WS and delirium. Therefore, an educational plan and sensibilization of health care professionals, particularly nurses, due to their central role in using monitoring tools, need to be implemented in the ICUs. 
Validated scales adopted in critically ill children to evaluate the level of analgosedation are:

- the CBS, a scoring system based on observational and behavioural variables, to evaluate distress (including pain) in critically ill children. $[43,44]$ The CBS indicates a range of adequate sedation and scores of over- and under-sedation. It may be used also in post-surgical ventilated children, including cardiac patients. The ESPNIC strongly recommends adopting this tool to monitor every 4-8 hours the level of sedation in critically ill children admitted to ICU.

- the State Behavioural Scale, a 6-level scoring system to evaluate patient's level of sedation with respect to his/her planned goal. [45]

- the Richmond Agitation-Sedation Scale, a 10-level scoring system to evaluate patient's level of sedation with respect to his/her planned goal.[46]

- the FLACC (Face, Legs, Activity, Cry, Consolability), the WONG BAKER and the Numeric Rating (NRS) scales used (depending on patient's ages) to specifically evaluate and monitor pain. $[47,48,49]$

Regular use of these scales may increase workload but promotes the improvement of quality of care.

\section{RECOMMENDATION 3:}

We recommend regularly monitoring with validated tools the level of analgosedation of pediatric patients admitted to ICU.

Strength of recommendation: Strong

\section{li. Difficult Analgosedation}

At present, no unique criteria exist to define difficult analgosedation. Recently, Lebet et al. conducted a survey between experts, to design a model to predict difficult analgo-sedation, dividing associated variables in three groups. [50]

Variables related to sedation: need touse more than three sedative drugs, presence of inadequate sedation lasting more than 2 hours, need to increase sedative doses higher than $90^{\circ}$ centiles considering usual starting dose, need to administer to the patient intermittent doses of neuromuscular blocking agents to adapt him/her to ventilator.

Variables related to adverse events: suspected delirium,unplanned extubation,unplanned removal of invasive devices, paradoxical response to sedation.

Demographic/diagnostic variables: 21 trisomy, previous sedations, non-communicating patients, Body Mass Index higher than $90^{\circ}$ centile, cancer disease, moderate disability, bronchiolitis. 
Some of these variables were reported by Mencia et al, adding the presence of prolonged mechanical ventilation. [51]

\section{WHICH ANALGOSEDATIVE DRUGS ARE USEFUL IN DIFFICULT ANALGOSEDATION?}

In difficult analgodsedation it is indicated to use third-line drug strategy, including molecules acting on GABA or N-methyl-D-aspartate (NMDA) receptor, with possible risks of neurotoxicity.

Ketamine continuous infusion may have a role as an adjuvant to children difficult to sedate. Nowadays, prospective studies on ketamine in PICU are lacking. [52] The review of Golding et al, including only case report series, reported the achievement of satisfactory sedation and analgesia by using ketamine, with an improvement of pulmonary compliance and oxygenation, and minimal adverse effects (nystagmus, flushing, one report of hypertension). [53]

Ketamine showed a good safety profile particularly in patients with bronchospasm, thanks to bronchodilator effects of this drug.[54] A retrospective study published in 2017 reported that ketamine may reduce the development of opioid tolerance. [55] In a second retrospective study published in 2019, ketamine, administered in continuous infusion, didn't impact mortality and haemodynamic stability of critically ill children. [56]

Propofol, according to Italian Medical Agency (AIFA) recommendations, should not be used in pediatric ages in continuous infusion, particularly due to the risk of development Propofol Infusion Syndrome. Nevertheless, a dose less than $4 \mathrm{mg} / \mathrm{Kg} / \mathrm{hour}$ has been reported safe if the infusion lasts no more than 48 hours. [57]

Sevoflurane has been used in PICU as an adjuvant to morphine infusion, with the AnaConDa system, obtaining weaning from other sedatives in most patients. [50] A recent prospective study confirmed its efficacy in weaning from sedation and ventilation in critically ill children ready to extubation but very agitated and affected by WS. [58] Nowadays, the most effective dose and tolerated duration of treatment are not clear.

Few studies exist on the efficacy of levomepromazine as a sedative agent in pediatric population admitted to ICU. At present, it could be used in agitation states refractory to other treatments. $[59,60]$

\section{RECOMMENDATION 4:}

In difficult analgesia/sedation we suggest using ketamine, due to its good safety profile.

Strength of recommendation: Weak

\section{lii. Neuromuscular Blocking Agents}


Neuromuscular blocking agents (NMBA) in critically ill children are limited to specific indications: to guarantee patient's immobility, to adapt patients to mechanical ventilation, to obtain a reduction of oxygen demand. Moreover, NMBA are used during the procedure of endotracheal intubation. NMBA are wieldy, show predictable pharmacokinetics a

Neuromuscular blocking agents (NMBA) in critically ill children are limited to specific indications: to guarantee patient's immobility, to adapt patients to mechanical ventilation, to obtain a reduction of oxygen demand. Moreover, NMBA are used during the procedure of endotracheal intubation. NMBA are wieldy, show predictable pharmacokinetics also in children and didn't report severe adverse effects. Moreover, sugammadex, an antagonist of rocuronium bromide with a good safety profile in infants and children, gives clinicians the possibility to obtain an immediate reversal effect.

e with a good safety profile in infants and children, gives clinicians the possibility to obtain an immediate reversal effect.

The Pediatric Cardiac Intensive Care Society doesn't report specific indications of using NMBA, but underlined that hyponatremia, hypokalemia, and hypocalcemia may increase their effect whereas hypermagnesemia may decrease it. [3]

The Pediatric Acute Lung Injury Consensus Conference recommends the use of NMBA in patients with Pediatric Acute Respiratory Distress Syndrome and adequately sedated, to adapt patients to mechanical ventilation, to decrease doses of sedative drugs, to obtain a reduction of oxygen demand and respiratory effort. [61]

A prospective study on children ventilated more than 24 hours and treated with NMBA in continuous infusion reported a reduction of the thickness of diaphragmatic muscle, but the clinical impact of these data needs to be confirmed. [62] A secondary analysis of an RCT on therapeutic hypothermia showed the use of NMBA doesn't impact mortality and morbidity of critically ill children. [63] A recent case-control study reported an increase in the incidence of infections in patients with acute kidney insufficiency treated NMBA. [64]

\section{RECOMMENDATION 5:}

We suggest using neuromuscular blocking agents in patients with severe respiratory insufficiency and persistent patient-ventilator asynchrony despite actions taken to limit the rate of asynchrony.

\section{Strength of recommendation: Weak}

WHICH IS THE ADEQUATE MONITORING OF ANALGOSEDATION IN PEDIATRIC PATIENTS ADMITTED TO ICU AND TREATED WITH NEUROMUSCULAR BLOCKING AGENTS?

In patients treated with NMBA, monitoring the level of sedation is a priority and a challenge. Patients are immobilized, thus observational scales cannot be used. In pediatric patients, the interruption of NMBA 
infusion with the aim to monitor patients with validated observational scales [61] may promote adverse events.

At present, continuous processed electroencephalogram (cpEEG) is the most used and studied tool in adults, but few studies exist in pediatric patients. An RCT performed in the Operating Room evaluated the effect of mivacurium on Bispectral Index and Cerebral State Index reporting no modification of the scores after NMBA infusion and suggesting the possibility to correctly monitor the level of sedation adopting cpEEG. [65] Even though that cpEEG is not validated to evaluate the level of sedation in pediatric patients (particularly infants) treated with NMBA and its availability is still limited, we suggest adopting this tool in critically ill patients if observational scales are not applicable. However, the correct use of cpEEG requires specific skills in analysing the trace and in understanding the score.

\section{RECOMMENDATION 6:}

We suggest monitoring the level of sedation with continuous processed EEG in patients treated with neuromuscular blocking agents, considering the limitation and the availability of the device.

\section{Strength of recommendation: Weak}

\section{Iv Sleep}

Characteristics of sleep change with age and neurological development, both in duration than in frequency of a phase respect to the others. [66]

ICU admission exposes patients to environmental factors, procedures and drugs, causing a modification of physiologic phases of sleep. Most alterations of sleep architecture were reported in ventilated children. [67]

In burned pediatric patients admitted to ICU, sleep modification was frequently reported. $[68,66]$ Pain, fear, anxiety, often present in burned patients, promote sleep negative effects, especially in younger children. Most analgesic and sedative drugs (opiates, benzodiazepine, ketamine, propofol) reduce slowwave sleep phases of the Non-Rapid Eye Movement period and Rapid Eye Movement (REM) period, whereas stage 1 increases, with frequent arousals.[66, 69, 70]

Sleep modifications develop in ICU and persist after patient's discharge. [71]Alterations of sleep architecture or sleep duration impact many physiologic mechanisms promoting delirium or neurocognitive/neuro-psychiatric long-term sequelae, immunodepression, metabolism and respiratory insufficiency, thus preventing patient's recovery and patient's weaning from mechanical ventilation.

Despite the evidence of a negative impact on patient's outcome caused by sleep modifications, only recent studies covered this research area in pediatric population admitted to ICU, also due to the presence of many confounding factors in this setting. Moreover, different tools to evaluate the quality of sleep 
exist: polysomnography (PSG), actigraphy, cpEEG and methods based on the observation of patient's behaviour.

PSG is the gold standard. However, this tool is not diffused in ICU and requires high competence to be used. Studies performed with PSG reported that structure of sleep is frequently modified in ICU: 1) time to achieve the first phase of sleep is longer (Sleep Latency), 2) duration of sleep (Total Sleep Time) doesn't change but covers mostly the daytime hours, 3 ) duration of deepest phases (stage 3, Slow-Wave Sleep), and REM phase, decreases; 4) duration of less deep phases (stage 1) increases; 5) arousals increases. [72] Moreover, in critically ill children EEG waves may be modified by the "brain injury" due to the clinical status and due to administered drugs. Finally, NMBA infusion makes PSG impossible to record patient's muscular activity, required to analyse the electro-oculogram trace. [67]

Actigraphy is a less complex tool respect to PSG. However, it has not been validated in critically ill patients. It may be influenced by every movement (i.e. nursing procedure). Finally, actigraphy doesn't evaluate the quality of sleep.

cpEEG shows a good correlation with PSG, except for the possibility to identify alert states (Wakefulness), periods after sleep onset (Wakefulness After Sleep Onset) and REM phases. [73]

Methods based on the observation of patient's behaviour often don't correlate with PSG. $[74,67]$ WHICH IS THE BEST STRATEGY TO OPTIMIZE SLEEP IN PEDIATRIC PATIENTS ADMITTED TO ICU?

Pharmacologic treatment (melatoni and dexmedetomidine) to prevent and treat sleep alterations in ICU is not supported by evidence-based proof, in pediatric age.

In adults, some studies reported a positive effect of non-pharmacologic strategies, such as environmental modifications (particularly light control), on the suprachiasmatic nucleus and circadian rhythm. $[75,76,77]$

In neonates, non-pharmacologic treatment, like the use of nests, hammocks, gentle touch, handling, protocols based on noise reduction and light control, parental involvement in patient's care, showed an evidence-based efficacy on quality of sleep. [78] It is reasonable that also in pediatric patients these strategies might produce the same results.

Finally, some positive effects on duration and quality of sleep were reported using sequentially haloperidol and zolpidem on pediatric burned patients. [79]

\section{RECOMMENDATION 7:}

We recommend adopting in all pediatric patients admitted to ICU strategies to prevent sleep alterations, particularly non-pharmacologic ones (relaxing techniques, parental involvement, control of environmental factors).

Strength of recommendation: Strong 


\section{Delirium}

Delirium is an acute cerebral condition complicating outcome in critically ill children. According to Diagnostic and Statistical Manual of Mental Disorders Fifth Edition (DSM-5) by the American Psychiatric Association, delirium presents 5 key features: a disturb of consciousness and "awareness", acute onset (hours to days) with a fluctuating course during the day, presence of other cognitive deficit (memory, language, visive-spatial, perceptive), criteria 1 and 3 are not dependent on pre-existing neurocognitive deficits or on a severe awareness deficit (like coma), evidence (history, physical examination, laboratory tests) that delirium is a consequence of a clinical condition, a substance intoxication, weaning from drugs, toxin or multifactorial factors. [80, 81, 21]

The physiopathology of delirium is complex. Three mechanisms seem to be particularly involved: neuroinflammation, modification of neurological mediators due to drugs administration, oxidative stress due to a clinical condition. The final result is a modification of neurological transmission with integration/processing of sensory inputs and motorial response.

In critically ill children three factors contribute to delirium development: clinical condition, pharmacological treatments, environmental factors. $[83,84]$

Three subtypes of pediatric delirium exist: hyperactive delirium (characterized by agitation, restlessness, hypervigilance, combative behaviour), hypoactive delirium (characterized by lethargy, deficit of attention, decreased response to stimulus), mixed delirium (characterized by hyperactive and hypoactive aspects). Hypoactive delirium is the most frequent subtype. It is correlated with a worse outcome. In a longitudinal study on pediatric delirium, hypoactive and mixed delirium were the most frequent subtypes $(46,4 \%$ and $45,2 \%$ respectively) whereas hyperactive one was present in only $8,4 \%$ of patients. Children with delirium often show a modification of psychomotor activity, with delay in response to a stimulus or continuous agitation; moreover, emotional lability, inconsolable status, excessive quietude are present.

Frequently, the onset of pediatric delirium is during the first three days of ICU admission. [85, 82, 86, 87] Recent studies reported delirium in more than $20 \%$ of ICU admission. In a multicentric paper on 25 PICUs, the general prevalence of delirium was $25 \%, 53 \%$ in ventilated children. The highest prevalence was reported in critically ill children affected by inflammatory/infective diseases. [88]

Finally, it must be considered that the presence of delirium increases costs of PICU admission, independently from duration of PICU admission, patient's severity and age.[89]

The presence of delirium promotes complications in pediatric critically ill patients. A prospective study showed a strong and independent association between pediatric delirium and mortality. [85] A previous study reported that pediatric delirium increases duration of ventilation and length of ICU stay. [90] Nowadays, no evidence exists on the presence of long-term cognitive disorders in children with a diagnosis of delirium during ICU admission. However, literature is scarce and the impact on caregivers has not been studied. [91] 
Non -modifiable risk factors for the development of pediatric delirium are: pre-scholar age, mechanical ventilation, cognitive deficit, congenital cardiopathy, hepatic insufficiency, the severity on admission, treatment with vasopressors or with antiepileptic drugs, ICU stay longer than 5 days. $[92,85,89,93,86$, 87] At present, other potential factors (burns, transfusions) need to be confirmed.

Modifiable factors for the development of pediatric delirium are: treatment with benzodiazepines (it increases from 2.5 up to 5 times the risk of developing delirium, with dose-dependent effect), use of restraint and patient's immobilization, presence of noise, presence of light, modification of sleep- awake rhythm, absence of parents during ICU stay, treatment with anticholinergic drugs. [85, 23, 82, 87, 22] For this reason, preventive bundles for delirium are proposed with to reduce its incidence in critically ill children. (Figure 2) However, their implementation may be hindered by structural problems or lack of resources.

\section{RECOMMENDATION 8:}

We recommend working on modifiable risk factors, particularly reducing the use of benzodiazepines.

\section{Strength of recommendation: Strong}

\section{WHICH IS THE BEST STRATEGY TO USE IN PEDIATRIC DELIRIUM?}

The pivot of strategy is represented by the identification and treatment of risk factors. Moreover, modifiable factors need to be considered and minimized. [43, 95]

Great attention should be paid to create a familiar and comfortable environment, with light and noise reduction. Strategies to prevent delirium are usually displayed in "bundles". $[96,97]$ If a patient presents delirium despite the preventive strategies, bundles application should be maximized.

Pharmacologic treatment follows adult therapies, due to the paucity and low quality of literature in pediatric age. Antipsychotic drugs, off-label for indication and, sometimes, age, in children may be used in selected cases. An electrocardiogram needs to be checked before treatment, particularly QT interval. A multidisciplinary approach is suggested, involving a neurologist or neuropsychiatrist.

These drugs are contraindicated if other molecules prolonging QT are administered to the patient or if patient suffers of severe cardiopathy or heart block. Finally, electrolytes should be regularly assessed. [98]

According to a recent study, haloperidol reported adverse events even if the dosage was correct and blood level was below the therapeutic range, due to the occupation of dopaminergic receptor D2. [99] Olanzapine, risperidone e quetiapine has been used in pediatric delirium with good efficacy in low-quality studies (retrospective ones, case series). [100, 101, 102] 
Promising but anecdotal studies suggest a role of dexmedetomidine in the treatment of pediatric delirium. [103]

\section{RECOMMENDATION 9:}

We suggest basing the treatment of pediatric delirium on maximizing preventive bundles. Antipsychotic drugs may be used with careful consideration of contraindications.

\section{Strength of recommendation: Moderate}

WHICH IS THE ADEQUATE MONITORING OF DELIRIUM IN PEDIATRIC PATIENTS ADMITTED TO ICU?

At present, only a third of PICUs adopt tools to monitor delirium [104], despite validated scales exist. In 2011, following the Confusion Assessment Method for Intensive Care Unit (CAM-ICU) for adults, the Pediatric Confusion Assessment Method for the Intensive Care Unit (pCAM-ICU) was developed to monitor delirium in children older than 5 years. [105] In 2014, the Cornell Assessment of Pediatric Delirium (CAPD) was validated, including one year later "anchor points" to make the diagnosis of delirium in younger age and in children with developmental delay. $[106,107]$ To be applicable in the same groups of patients, in 2016 the Preschool Confusion Assessment Method for the ICU (psCAM-ICU), was developed. [108]

In 2018 the Sophia Observation Symptoms-Pediatric Delirium (SOS-PD) scale was derived from the Sophia Observation withdrawal Symptoms scale (SOS); to monitor with a single tool both withdrawal syndrome and, thanks to the inclusion of items related to the cognitive and behavioural status, delirium. This scale may be applied in children older than 3 months. [109]

All tools for the diagnosis of delirium include signs and symptoms of WS. Therefore, despite literature reported the presence of WS and delirium in the pediatric population, the overlap of signs and symptoms makes it difficult to distinguish between the two entities. [110]

ESPNIC recommends adopting CAPD to monitor pediatric delirium and to educate health professionals working in PICU to identify it.[4]

\section{RECOMMENDATION 10:}

We recommend regular monitoring delirium in critically ill children every day of the ICU stay, using validated tools.

Strength of recommendation: Strong

\section{Vi Withdrawal Syndrome}

Prolonged infusion of opiates and benzodiazepines, through pharmacodynamic mechanisms of receptor desensitization and up-regulation of excitatory intracellular pathways, promotes tolerance. Tolerance is 
the need to increase doses to obtain a therapeutic effect. It is associated with dependence, that is the need to administer a substance to avoid withdrawal symptoms. WS is the set of symptoms due to an abrupt stop or a too fast weaning from drugs inducing tolerance and/or dependence. It is characterized by central neurologic system, gastrointestinal and autonomic nervous system signs.

The incidence of opiates and benzodiazepine WS varies between $22,6 \%$ and $64,6 \%$. [111, 112] This high incidence was reported in a multicentre prospective study performed in Italian PICUs. [113]

WS developed after prolonged dexmedetomidine infusion overlaps symptoms of WS after opiates and benzodiazepines treatment. $[114,115]$

Patients with WS present a higher duration of mechanical ventilation and a longer ICU and hospital stay. At present, no studies exist on long-term neurocognitive sequelae after the development of WS. [116, 113] IS IT IMPORTANT TO WORK ON RISK FACTORS FOR PEDIATRIC WS IN ICU?

The most known risk factors for pediatric WS are drugs duration of infusion and administered cumulative doses. $[117,118]$ However, some studies reported the development of WS after infusion lasted less than 5 days. $[111,112]$

WS due to dexmedetomidine is reported after 48 hours of infusion and no data exist on how to wean from this drug. [115]

Opiates cumulative doses (including supplemental boluses) equal to or higher than $166,7 \mathrm{mg} / \mathrm{kg}$ (morphine equivalent) and benzodiazepines cumulative doses equal or higher than $60 \mathrm{mg} / \mathrm{kg}$ (midazolam equivalent) are considered at risk for WS. Recent studies reported, particularly for midazolam, the highest administered doses $(0,3-0,4 \mathrm{mg} / \mathrm{Kg} / \mathrm{hour})$ as predictive for the development of WS. [117, 111,112]

Moreover, some modifiable risk factors were identified: the choice of drug (morphine seems to show a lower probability of risk than other opiates), adoption of multiple sedative drugs, daily weaning higher than $20 \%$ of the initial dose, high nurse workload. At present, demographic (youngest age) and clinical (neurocognitive delay) variables are not considered always risk factors of WS. $[113,119,112,111,120]$

\section{RECOMMENDATION 11:}

\section{We recommend to working on modifiable risk factors of WS, particularly avoiding weaning higher than a daily reduction of $20 \%$ respect on the initial dose.}

\section{Strength of recommendation: Strong}

\section{WHICH ARE THE BEST STRATEGIES IN PEDIATRIC WS?}

The development of WS needs to be considered during all the analgesic and sedative treatment to minimize risk factors and work on modifiable ones. 
Considering prevention, some studies reported the efficacy of nurse-driven protocols in reducing cumulative doses, particularly of benzodiazepines, through increased use of validated monitoring tools. [34, 37, 35]According to some authors, the use of alpha agonists may reduce analgesic and sedative cumulative doses. [121, 122]

As first-line strategy of treatment, the modulation of the weaning plan should be implemented, eventually using additional boluses of the drug considered responsible for the symptoms.

Molecules adopted in neonatal age to prevent or treat WS are not considered evidence-based strategies in pediatric age. [114] No evidence-based data exist to support the role of receptor antagonists, like naloxone and dextromethorphan. Methadone, a synthetic opioid, may be used in opioid weaning with the aim of stopping the administered intravenous opiate in 24 hours. Excessive sedation is the most frequent adverse effect of this drug. $[123,124]$

At present, the role of alpha agonists in treating WS needs to be confirmed. However, both clonidine as dexmedetomidine might have a potential action in preventing and treating WS, due to an opioid-sparing action mechanism and suppression of catecholamine release. [125,126]

\section{RECOMMENDATION 12:}

We recommend treating withdrawal symptoms with additional boluses of the drug considered to be responsible for the symptoms and modifying the weaning plan.

Strength of recommendation: Strong WHICH IS THE ADEQUATE MONITORING OF WS IN PEDIATRIC PATIENTS ADMITTED TO ICU?

Diagnosis of WS and evaluation of the efficacy of its treatment are challenging in ICU, due to the overlapping of symptoms of inadequate analgesia or sedation, delirium, discomfort induced by environmental factors (noise, light...), or other pathological conditions. Moreover, opiates and benzodiazepines withdrawal symptoms overlap. [110]

ESPNIC identified two monitoring tools for opiates and benzodiazepine WS, validated in critically ill children:

- WAT-1

- sos

WAT-1 was the first developed tool, validated in pediatric age. WAT-1 and SOS showed similar sensibility and specificity, whereas modality of application, numbers of items, and patient's stimulation are different. $[4,127,128,129]$ 
We recommend regular monitoring withdrawal symptoms in critically ill children treated with analgesics and/or sedatives longer than 72 hours, adopting validated tools.

Strength of recommendation: Strong

\section{Vii Pediatric Palliative Sedation}

PPS is defined the intentional administration of sedative drugs to alleviate one or more refractory symptoms, with the aim of reducing the child's awareness at the end of his/her life. [130] A symptom is refractory when any possible treatment fails or if no method is available to alleviate it in a reasonable time a dying child may tolerate. [131] The most common refractory symptoms at the end of life in pediatric age are: pain, dyspnoea, fatigue, delirium, seizures, vomit. [132]

Definition of PPS doesn't intentionally distinguish between continuous and intermittent sedation, between light and deep sedation, considering the "proportionality" the ethical pivotal rationale. [133]

The aim of PPS isn't to promote death during the terminal phase of life, but to alleviate pain and distress due to refractory physical symptoms if no other therapeutic option exists. [134]

\section{WHICH ARE THE ORGANIZATION STRATEGIES TO PERFORM AN ADEQUATE PPS?}

To care and to plan the end of life are crucial, not only for the child but also for parents. Parents will keep in their mind last the period of his/her child's life forever.

A clear and multidisciplinary plan of care must be established. It needs to be agreed with parents after informed consent, indicating to stop/not to start new supporting therapies with to permit the natural patient's death. All the figures caring the child need to be involved: paediatricians, intensivists, palliativists, neonatologists, oncologists, pneumologists, neurologists, neuropsychiatrists, psychologists, social workers $[135,136,134]$

At present, most incurable children die in ICU, despite the fact this setting is not appropriate for many reasons. $[137,138]$

In Italy, law 38/2010 and 219/2017 declares as human right the possibility, if refractory symptoms are present, to receive palliative sedation, making PPS is accepted from an ethical point of view. $[139,140]$

\section{RECOMMENDATION 14:}

We recommend performing PPS early defining an interdisciplinary plan agreed with parents.

Strength of recommendation: Strong

WHICH IS THE THERAPEUTIC STRATEGY TO OBTAIN AN ADEQUATE PPS? 
At present, a unique therapeutic strategy to conduct PPS doesn't exist, but recommendations of experts and single centres protocols are reported. [141]

If the patient shows extreme agitation, anxiety, or dyspnea, the use of midazolam associated with morphine is considered in many publications the first-line strategy, adopting variable doses. [142]Morphine is used as a single drug in $25 \%$ of cases and associated with midazolam in $50 \%$. [143]

As second-line or third-line therapy, neuroleptics, barbiturates, propofol and recently dexmedetomidine were administered if midazolam was not effective or when delirium is the refractory symptom to treat. [103] Some experts in this setting recommend the use of haloperidol, cited in WHO 2008 list of necessary drugs in pediatric palliative care, to reduce midazolam. $[144,134]$ Single centres experience reported as effective the use of propofol particularly in adolescents or if the first-line strategy was ineffective. Doses of propofol reported in the literature are in the range between 0,5 and $16 \mathrm{mg} / \mathrm{Kg} / \mathrm{h}$; duration of infusion has been described up to 30 days. $[130,145,146,147,148]$ Ketamine has been used in children to control severe pain, with the aim of decreasing opioids escalation or reducing neuropathic pain. $[149,150,151]$

The pivotal is to obtain patient's comfort, increasing doses of drugs and evaluating their efficacy. Analgesic and sedative doses in PPS are generally higher respect to them reported in the summary of product characteristics. Vital sings monitoring during PPS is not recommended. [131]

\section{RECOMMENDATION 15:}

We suggest adopting a personalized strategy to achieve PPS in children, to ensure the maximal efficacy using dosed tailored to the patient.

Strength of recommendation: Weak

\section{Viii Analgesia And Sedation In Pediatric Patients With Neurodevelopmental Delay}

In children with neurodevelopmental delay, pain response is often a maladaptive behaviour, with freezing, self-injury or modification of the usual daily skills. [152]

WHICH IS THE ADEQUATE MONITORING OF ANALGESIA AND SEDATION IN PEDIATRIC PATIENTS WITH DEVELOPMENTAL DELAY ADMITTED TO ICU?

Pain assessment is difficult due to the patient's comorbidities and the level of neurodevelopmental delay, resulting in different expression and communication modalities. [153] Patients with cerebral palsy often show movement and posture abnormalities with seizures, muscular problems, sensorial, cognitive, communicative or behavioural disorders. Therefore, the risk of underestimating the severity of pain or delaying the diagnosis in these patients is high, also due to the presence of many environmental disturbing factors. A recent secondary analysis of a randomized study reported, in children with 
developmental delay admitted to ICU, the use of a lower cumulative dose of analgesic and sedative drugs compared to the other patients. However, a higher incidence of WS was present. The authors underlined the risk of adopting inadequate monitoring tools to evaluate the discomfort in these children. [154]

Revised Face, Legs, Activity, Cry, Consolability (r-FLACC) and Non-Communicating Children's Pain Checklist Postoperative Version (NCCPC-PV) are validated scales for children with developmental delay. $[155,156]$ They are easily usable in the hospital also without the presence of caregivers. The Italian version is available $[157,158]$ but no validation in ICU exists. During the evaluation, the involvement of caregivers is necessary to better understand child's behavioural and its modifications. More complex tools (NCCPC-revised, Paediatric Pain Profile) are diary-dossier of child's history of pain with personalized scores to evaluate the severity of that patient's pain during an event. These scales are not applicable in ICU. Finally, CBS was proposed to evaluate distress in children ages 0-3 years with Down Syndrome, with good results. [159]

To monitor delirium, authors of CAPD scale suggested the development of personalized "anchor points" for children with developmental delay, using caregivers' collaboration during ICU admission. [107]

Instrumental monitoring may be not accurate in these children, because seizure, cerebral disease, antiepileptic/neurological therapy may modify the data, as reported in a study performed during surgery in children with developmental delay, showing lower BIS scores than other children. [160]

Patients with neurodevelopmental delay often need chronic drug treatment. Potential interactions between these therapies and analgesic and sedative drugs administered in ICU and their impact on patient's safety should be considered. [161] At present, studies on this issue are lacking.

\section{RECOMMENDATION 16:}

In children with developmental delay, we suggest adopting validated tools to monitor the level of sedation, the presence of delirium and withdrawal syndrome in ICU, considering their limitations and involving the caregivers.

\section{Strength of recommendation: Weak}

WHICH IS THE IDEAL COMMUNICATION TO PARENTS OF CRITICALLY ILL CHILDREN ADMITTED TO ICU IN TERMS OF ANALGESIA AND SEDATION?

\section{THE PROBLEM OF THE “OFF LABEL” ANALGESICS AND SEDATIVES}

In the pediatric age many drugs are administered considering the dose for adults. In these cases, no authorization from national or international Drug Agency exists. For this reason, studies are highly required to gain pharmacologic data in neonates and children. Unfortunately, at present many drugs are off-label in pediatric age. Ethical, economic, biologic and patients' physiologic factors make it difficult to promote studies related to this topic. Drug Agency developed skills and facilities to produce plans for 
pediatric research (PIPs). In 2017 the Committee report presented to Parliament and European Council, 10 years after UE Pediatric Regulation, showed an increased number of approved PIPs and authorizations for pediatric drugs, today "in label". [162] Looking at European EudraCT database, clinical research increased, but a new approach to research, an alternative to clinical trials due to paucity of the sample (i.e. simulation models, extrapolation models...) needs to be developed.

In Italy, two laws regulate off-label drugs in National Health System (NHS). The first one is 648/1996 law, related to drugs used for not authorized indications if an alternative option is not available. The other law is $79 / 2014$, related to drugs used for not authorized indications if an alternative option is available, when scientific data reported an economic advantage and appropriateness. Thank to this regulation, after the official request from SARNePI, many analgesic and sedative drugs were authorized by AIFA: ketamine, morphine, fentanyl, alfentanil, remifentanil, propofol, midazolam, neuromuscular blocking agents, local anesthetics, antagonists like sugammadex, naloxone and flumazenil and, more recently, dexmedetomidine. $[163,26]$ For each drug a specific authorized indications for the use were specified.

\section{PARENTS' OPINION}

Parents' involvement is a priority for pediatric patients admitted to ICU. The approach to family, according to the model of family-centred care, is an efficient and always usable tool. Giving parents understandable information describing "what's happening", and "what the child will feel", eventually using a cultural mediator, listening to parents' perspective and point of view, respecting, if possible, parents' desires and promoting parents' participation in the patient's care are necessary steps. Recent studies particularly underlined the importance of parents' role in the prevention, evaluation and treatment of delirium [164] and WS . [165]

To evaluate parents' opinions, in these recommendations we adopted an anonymous questionnaire with 6 questions related to analgesia and sedation. It was administered in 4 PICUs to 19 parents, during the discharge phase. Their child needed to be admitted longer than 48 hours and analgesia and sedation needed to be administered during PICU stay.

Considering results (see below), satisfaction was good in $4 / 6$ topic (questions $1,2,3,5$ ) and in 18/19 questionnaires.

Two areas of improvement were reported:

- Environment, considered disturbing in terms of lights, noise, interruptions (question 4) (Figure 3)

- Communication related to WS and, particularly, delirium, considered inadequate (question 6) (Figure 4)

\section{RECOMMENDATION 17:}

We recommend explaining to parents the meaning of analgesia and sedation and off-label drugs. If analgesia and sedation lasted more than 48 hours, we recommend informing parents about the risk of 
withdrawal syndrome and delirium development.

Strength of recommendation: Strong

\section{Conclusions}

In the pediatric age, the literature covering analgesia and sedation is poor and the quality of the studies is quite low. However, some relevant information has recently emerged. First, the indication to reduce the use of benzodiazepine as the first-choice sedative agent, preferring the alpha agonists, as an effective and safe option, associated with the opiates to cover the pain. Moreover, assessment is a key point to ensure the best care to patients, to maintain an adequate level of comfort and particularly to identify delirium and withdrawal syndrome. Four special aspects are developed in this document. The approach to patients with a development delay, which requires appropriate tools to be appropriately evaluated in terms of pain and sedation. The attention to the quality of patients' sleep, not only to decrease the incidence of delirium. The significance of palliative sedation and the best strategy to achieve it in the PICU setting. Finally, the importance of complete communication to parents to explain the meaning of analgesia and sedation and its risks.

The list of research recommendations (Table 1) emphasizes how much it remains to be done. However, following shared recommendations remains the first step to offer the best analgesia strategy and sedation for critically ill children.

\section{Abbreviations:}

AMIETIP Medical and Nursing Academy of Paediatric Emergency and Intensive Care

ANIARTI National Association of Critical Care Nurse

ESPNIC European Society of Pediatric and Neonatal Intensive Care

CAPD Cornell Assessment Pediatric Delirium

CBS Comfort Behaviour Scale

FLACC (Face, Legs, Activity, Cry, Consolability

GABA aminobutyric acid

ICU Intensive Care Unit

NMBA Neuromuscular blocking agents

PICO population (P), intervention (I), control (C) and outcomes (O) 
PICU Pediatric Intensive care Unit

PPS pediatric palliative sedation

PSG polysomnography

REM Rapid Eye Movement

RCT randomized controlled trials

SIAARTI Italian Society of Analgesia Anesthesia and Intensive Care

SARNePI Italian Society of Neonatal and Pediatric Anesthesia and Intensive Care

SOS Sophia Observation withdrawal Symptoms scale

WAT-1 Withdrawal Assessment Tool -1

WS iatrogenic withdrawal syndrome

\section{Declarations}

\section{Funding:}

Participation in the recommendation process was voluntary and not paid. The project was not funded.

Conflict of interests:

The authors declare that they have no competing interests.

Availability of data and material

Data and materials are available within the article and the Supplementary materials

Code availability

Not applicable

Authors' Contributions

Dr. Amigoni and Dr. Mondardini conducted the project and take, selected the research questions, made the literature research, analysed selected article, drafted the manuscript, developed the parents' questionnaire, discussed the recommendations. Prof Conti discussed the recommendations, performed a critical review. Dr Conio, Dr Corno, Dr Ferrero, Dr Giugni, Dr L’Erario, Masola, RN, Dr Moliterni, Dr Zito Marinosci selected the research questions, made the literature research, drafted the manuscript, discussed the recommendations. Fazio, RN made the literature research, drafted the manuscript, 
developed the parents' questionnaire, discussed of recommendations. Dr Gentili made literature research, drafted the manuscript. Dr Ricci, Dr Vasile, Dr Romagnoli selected the research questions, made the literature research, analysed the selected articles, drafted the manuscript, discussed the recommendations. Dr Pagano, Dr Vitale selected the research questions, developed the parents' questionnaire, discussed the recommendations. All authors performed a critical revision of the manuscript for important intellectual content and approved the final version.

\section{Ethical approval}

Not applicable. Not required

Consent to participate

Not applicable

Consent for publication:

All authors gave the consent

\section{Acknowledgment:}

We thankGaetano Cantalupo, University Hospital, Verona; Andrea Cortegiani, Palermo University, Palermo; Maria Grazia Foschino Barbaro, Pope Giovanni XIII Pediatric Hospital, Bari; Stefano Pro, Bambino Gesù Hospital, Roma.

We thank the following Scientific Societies for having endorsed these recommendations: the Italian Neonatal and Pediatric Society of Anesthesia and Intensive Care (SARNePI), the Italian Society of Analgesia Anesthesia and Intensive Care (SIAARTI), the Medical and Nursing Academy of Paediatric Emergency and Intensive Care (AMIETIP), the National Association of Critical Care Nurse (ANIARTI).

\section{References}

1. Playfor S, Jenkins I, Boyles C et al (2006) Consensus guidelines on sedation and analgesia in critically ill children. Intensive Care Med 32: 1125-1136.

2. Mondardini MC, Vasile B, Amigoni A et al (20149 Update of recommendations for analgosedation in pediatric intensive care unit. Minerva Anestesiol 80:1018-1029.

3. Lucas SS, Nasr VG, Ng AJ et al (2016) Pediatric Cardiac Intensive Care Society 2014 Consensus Statement: Pharmacotherapies in Cardiac Critical Care: Sedation, Analgesia and Muscle Relaxant. Pediatr Crit Care Med 17:S3-S15.

4. Harris J, Ramelet AS, van Dijk M et al (2016) Clinical recommendations for pain, sedation, withdrawal and delirium assessment in critically ill infants and children: an ESPNIC position statement for healthcare professionals. Intensive Care Med 42:972-986. 
5. Higgins JPT, Green S. Cochrane handbook for systematic reviews of interventions version 5.1.0 [updated March 2011]. Edited by: [http://www.cochrane-handbook.org]

6. Wells G, Shea B, O'Connell D et al (2013) The Newcastle-Ottawa Scale (NOS) for assessing the quality of non-randomised studies in metaanalyses. http://www.ohri.ca/programs/clinical_epidemiology/oxford.asp

7. American Academy of Pediatrics Steering Committee on Quality I, Management (2004) Classifying recommendations for clinical practice guidelines. Pediatrics 114:874-877.

8. Browers MC, Kho ME, Browman GP et al for the AGREE next steps Consortium (2010) AGRE II: advancing guideline development, reporting and evaluation in health care. CMAJ 182: E839-E842

9. Gatchel RJ, Reuben DB, Dagenais S et al (2018) Research agenda for the prevention of pain and its impact: report of the work group on the prevention of acute and chronic pain of the Federal Pain Research Strategy. J Pain 19:837-51.

10. Baarslag MA, Allegaert K, Knibbe CA et al (2017) Pharmacological sedation management in the paediatric intensive care unit. J Pharm Pharmacol 69:498-513.

11. Vet NJ, Ista E, de Wildt SN et al (2013) Optimal sedation in pediatric intensive care patients: a systematic review. Intensive Care Med 39:1524-1534.

12. Yu EH, Tran DH, Lam SW et al (2016) Remifentanil tolerance and hyperalgesia: short-term gain, longterm pain? Anaesthesia 71:1347-1362.

13. Welzing L, Oberthuer A, Junghaenel S et al (2012) Remifentanil/midazolam versus fentanyl/midazolam for analgesia and sedation of mechanically ventilated neonates and young infants: a randomized controlled trial. Intensive Care Med 38:1017-1024

14. Matic M, de Wildt SN, Tibboel D et al (2017) Analgesia and opioids: a pharmacogenetics shortlist for implementation in clinical practice. Clin Chem 63:1204-1213.

15. Horvat CM, Au AK, Conley YP et al (2017) ABCB1 genotype is associated with fentanyl requirements in critically ill children. Pediatr Res 82:29-35.

16. Zuppa AF, Benitez GR, Zane NR et al (2019) Morphine dose optimization in critically ill pediatric patients with acute respiratory failure: a population pharmacokinetic-pharmacogenomic study. Crit Care Med 47:e485-e494.

17. Zuppa AF, Conrado DJ, Zane NR et al (2019) Midazolam dose optimization in critically ill pediatric patients with acute respiratory failure: a population pharmacokinetic-pharmacogenomic study. Crit Care Med 47:e301-e309.

18. Vittinghoff M, Lönnqvist PA, Mossetti V et al (2018) Postoperative pain management in children: Guidance from the pain committee of the European Society for Paediatric Anaesthesiology (ESPA Pain Management Ladder Initiative). Paediatr Anaesth 28:493-506.

19. Tabacco B, Tacconi C, Amigoni A (2017) Survey on monitoring analgesia and sedation in the Italian Pediatric Intensive Care Units. Minerva Anestesiol 83:1010-1016. 
20. Andropoulos DB, Greene MF (2017) Anesthesia and developing brains - implications of the FDA warning. N Engl J Med 376:905-907.

21. Zuppa AF, Curley MAQ (2017) Sedation analgesia and neuromuscular blockade in pediatric critical care: overview and current landscape. Pediatr Clin North Am 64:1103-1116

22. Mody K, Kaur S, Mauer EA et al (2018) Benzodiazepines and development of delirium in critically ill children: estimating the causal effect. Crit Care Med 46:1486-1491.

23. Smith HA, Gangopadhyay M, Goben CM et al (2017) Delirium and benzodiazepines associated with prolonged ICU stay in critically ill infants and young children. Crit Care Med 45:1427-1435.

24. Kochaneck PM, Tasker RC, Carney N et al (2019) Guidelines for the management of pediatric severe traumatic brain injury, third edition: Update of the brain trauma foundation guidelines. Pediatr Crit Care Med 20:S1-S82.

25. Sperotto F, Mondardini MC, Vitale F et al (2019) Prolonged sedation in critically ill children: is dexmedetomidine a safe option for younger age? An off-label experience. Minerva Anestesiol 85:164172.

26. Mondardini MC, Astuto $M$, Amigoni A (2017) Inclusion of dexmedetomidine for pediatric indications in the list of medicinal products issued by the national law 648/96 Minerva Anestesiol 83:891-892.

27. Wolf A, McKay A, Spowart C et al (2014) Prospective multicenter randomized, double-blind, equivalence study comparing clonidine and midazolam as intravenous sedative agents in critically ill children: the SLEEPS (Safety profile, Efficacy and Equivalence in Paediatric intensive care Sedation) study. Health Technol Assess 18:1-212.

28. Hayden JC, Breatnach C, Doherty DR et al (2016) Efficacy of a2-agonists for sedation in pediatric critical care: a systematic review. Pediatr Crit Care Med 17:e66-e75.

29. Grant MJ, Schneider JB, Asaro LA et al (2016) Dexmedetomidine use in critically ill children with acute respiratory failure. Pediatr Crit Care Med 17:1131-1141.

30. Amula V, Vener DF, Pribble CG et al (2019) Changes in anesthetic and postoperative sedationanalgesia practice associated with early extubation following infant cardiac surgery: experience from the pediatric heart network collaborative learning study. Pediatr Crit Care Med 20:931-939.

31. Venkatraman R, Hungerford JL, Hall MW et al (2017) Dexmedetomidine for sedation during noninvasive ventilation in pediatric patients. Pediatr Crit Care Med 18:831-837.

32. Curley MAQ, Wypij D, Watson RS et al (2015) Protocolized sedation vs usual care in pediatric patients mechanically ventilated for acute respiratory failure: a randomized clinical trial. JAMA 313:379-389

33. Deeter KH, King MA, Ridling D et al (2011) Successful implementation of a pediatric sedation protocol for mechanically ventilated patients. Crit Care Med 39:683-688.

34. Neunhoeffer F, Kumpf M, Renk $\mathrm{H}$ et al (2015) Nurse-driven pediatric analgesia and sedation protocol reduces withdrawal symptoms in critically ill medical pediatric patients. Paediatr Anaesth 25:786794. 
35. Sanavia E, Mencía S, Lafever SN et al (2019) Sedative and analgesic drug rotation protocol in critically ill children with prolonged sedation: evaluation of implementation and efficacy to reduce withdrawal syndrome. Pediatric Crit Care Med 20:1111-1117.

36. Dreyfus L, Javouhey E, Denis A et al (2017) Implementation and evaluation of a paediatric nursedriven sedation protocol in a paediatric intensive care unit. Ann Intensive Care 7:36.

37. Gaillard-Le Roux B, Liet JM, Bourgoin P et al (2017) Implementation of a nurse-driven sedation protocol in a PICU decreases daily doses of midazolam. Pediatr Crit Care Med 18:e9-e17.

38. Neunhoeffer F, Seitz G, Schmidt A et al (2017) Analgesia and sedation protocol for mechanically ventilated postsurgical children reduces benzodiazepines and withdrawal symptoms-but not in all patients. Eur J Pediatr Surg 27:255-262.

39. Yaghmai BF, Di Gennaro JL, Irby GA et al (2016) A pediatric sedation protocol for mechanically ventilated patients requires sustenance beyond implementation. Pediatr Crit Care Med 17:721-726.

40. Gupta K, Gupta VK, Jayashree M et al (2012) Randomized controlled trial of interrupted versus continuous sedative infusions in ventilated children. Pediatr Crit Care Med 13:131-135.

41. Verlaat CW, Heesen GP, Vet NJ et al (2014) Randomized controlled trial of daily interruption of sedative in critically ill children. Paediatr Anaesth 24:151-156.

42. Vet NJ, de Wildt SN, Verlaat CW et al (2016) A randomized controlled trial of daily sedation interruption in critically ill children Intensive Care Med 42:233-244

43. Ista $E$, van Dijk M, Tibboel D et al (2005) Assessment of sedation levels in pediatric intensive care patients can be improved by using the COMFORT "behavior" scale. Pediatr Crit Care Med 6:58-63.

44. Boerlage AA, Ista E, Duivenvoorden HJ, de Wildt SN et al The Comfort Behaviour Scale detects clinically meaningful effects of analgesic and sedative treatment. Eur J Pain 19:473-479.

45. Curley MAQ, Harris SK, Fraser KA et al (2006) State Behavioral Scale: a sedation assessment instrument for infants and young children supported on mechanical ventilation. Pediatr Crit Care Med 7:107-114.

46. Kerson AG, De Maria R, Mauer E et al (2016) Validity of the Richmond Agitation-Sedation Scale (RASS) in critically ill children. J Intensive Care 4:65.

47. Voepel-Lewis T, Merkel S, Tait AR, et al (2002) The reliability and validity of the Face, Legs, Activity, Cry, Consolability observational tool as a measure of pain in children with cognitive impairment. Anesth Analg 95:1224-1229.

48. Garra G, Singer AJ, Breena RT et al (2010) Validation of the Wong-Baker Faces Pain Rating Scale in Pediatriuc Emergency Department patients. Acad Emerg Med 17:50-54.

49. Bijur PE, Silver W, Gallagher EJ (2001) Reliability of the visual analog scale for measurement of acute pain Acad Emerg Med 8:1153-1157.

50. Lebet RM, Asaro LA, Zuppa AF et al (2018) Face and content validity of variables associated with the difficult-to-sedate child in the paediatric intensive care unit: A survey of paediatric critical care clinicians. Aust Crit Care 31:167-73. 
51. Mencia S, Palacios A, Garcia M et al (2018) An exploratory study of sevoflurane as an alternative for difficult sedation in critically ill children. Pediatr Crit Care Med 19:e335-e341.

52. Walker T, Kudchadkar SR (2019) Pain and sedation management: 2018 update for the Rogers' textbook of Pediatric Intensive Care. Pediatr Care Med 20:54-61.

53. Golding CL, Miller JL, Gessouroun MR et al (2016) Ketamine continuous infusions in critically ill infants and children. Ann Pharmacother 50:234-50.

54. Heiberger AL, Ngorsuraches S, Olgun G et al (2018) Safety and utility of continuous ketamine infusion for sedation in mechanically ventilated pediatric patients. J Pediatr Pharmacol Ther 23:44754.

55. Neunhoeffer F, Hanser A, Esslinger M et al (2017) Ketamine infusion as a counter measure for opioid tolerance in mechanically ventilated children: a pilot study. Paediatr Drugs 19:259-65.

56. Park S, Choi AY, Park E et al (2019) Effects of continuous ketamine infusion on hemodynamics and mortality in critically ill children. PLoS One 14:e0224035.

57. Koriyama H, Duff JP, Guerra GG et al (2014) Is propofol a friend or a foe of the pediatric intensivist? Description of Propofol use in a PICU. Pediatr Critical Care Med 15:e66-71.

58. Pavcnik M, Groselj Grenc M (2019) Sevoflurane sedation for weaning from mechanical ventilation in pediatric intensive care unit. Minerva Anestesiol 85:951-961.

59. van der Zwaan S, Blankespoor RJ, Wolters AM et al (2012) Additional use of methotrimeprazine for treating refractory agitation in pediatric patients. Intensive Care Med 38:175-176.

60. Snoek A, James P, Arenas-López S et al (2014) Levomepromazine for difficult sedation in pediatric intensive care. J Pediatr Intensive Care 3:53-57.

61. Pediatric Acute Lung Injury Consensus Conference Group (2015) Pediatric Acute Respiratory Distress Syndrome: Consensus recommendations from the Pediatric Acute Lung Injury Consensus Conference. Pediatr Crit Care Med 16:428-439.

62. Glau CL, Conlon TW, Himebauch AS et al (2018) Progressive diaphragm atrophy in pediatric acute respiratory failure. Pediatr Crit Care Med 19:406-411.

63. Chin KH, Bell MJ, Wisniewski SR et al (2015) Effect of administration of neuromuscular blocking agents in children with severe traumatic brain injury on acute complication rates and outcomes: a secondary analysis from a randomized, controlled trial of therapeutic hypothermia. Pediatr Crit Care Med 16:352-358.

64. Guess R, Vaewpanich J, Coss-Bu JA et al (2018) Risk factors of ventilator-associated events in a PICU. Pediatr Crit Care Med 19:e7-e13.

65. Weber F, Kriek N, Blussévan Oud-Alblas HJ (2010) The effect of mivacurium-induced neuromuscular block on Bispectral Index and Cerebral State Index in children under propofol anesthesia - a prospective randomized clinical trial. Paediatr Anaesth 20:697-703.

66. Kudchadkar SR, Aljohani OA, Punjabi NM (2014) Sleep of critically ill children in the pediatric intensive care unit: A systematic review. Sleep Med Rev 18:103-110. 
67. AL-Samsam RH, Cullen P. (2005) Sleep and adverse environmental factors in sedated mechanically ventilated pediatric intensive care patients. Pediatr Crit Care Med 6:562-567.

68. Gottschlich MM, Jenkins ME, Mayes T et al (1994) The 1994 Clinical Research Award. A prospective clinical study of the polysomnographic stages of sleep after burn injury. J Burn Care Rehabil 15:48692.

69. Kamdar BB, Needham DM, Collop NA (2012) Sleep deprivation in critical illness: its role in physical and psychological recovery. J Intensive Care Med 27:97-111.

70. Gottschlich MM, Mayes T, Khoury J et al (2011) The effect of ketamine administration on nocturnal sleep architecture. J Burn Care Res 32:535-40.

71. Wiclox ME, Lim AS, Pinto R et al (2018) Sleep on the ward in intensive care unit survivors: a case series of polysomnography. Intern Med J 48:795-802.

72. Devlin JW, Skrobik Y, Gélina C et al (2018) Clinical Practice Guidelines for the Prevention and Management of Pain, Agitation/Sedation, Delirium, Immobility, and Sleep Disruption in Adult Patients in the ICU. Crit Care Med 46: e825-e873.

73. Benissa MR, Khirani S, Hartley S et al (2016) Utility of the bispectral index for assessing natural physiological sleep stages in children and young adults. J Clin Monit Comput 30:957-963.

74. Armour AD, Khoury JC, Kagan RJ et al (2011) Clinical assessment of sleep among pediatric burn patients does not correlate with polysomnography. J Burn Care Res 32:529-534.

75. Danielson SJ, Rappaport CA, Loher MK et al (2018) Looking for light in the din: an examination of the circadian-disrupting properties of a medical ICU. Intensive Crit Care Nurs 46:57-63.

76. Bion V, Lowe AS, Puthucheary Z et al (2018) Reducing sound and light exposure to improve sleep on the adult intensive care unit: an inclusive narrative review. J Intensive Care Soc 19:138-146.

77. Fan EP, Abbott SM, Reid KJ et al (2017) Abnormal environmental light exposure in the intensive care environment. J Crit Care 40:11-14.

78. Lago P, Garetti E, Bellieni CV et al (2017) Systematic review of nonpharmacological analgesic interventions for common needle-related procedure in newborn infants and development of evidencebased clinical guidelines. Acta Paediatr 106:864-870.

79. Armour AD, Gottschlich MM, Khoury JC et al (2008) A randomized, controlled prospective trial of zolpidem and haloperidol for use as sleeping agents in pediatric burn patients. J Burn Care Res 29:238-247.

80. Daoud A, Duff JP, Joffe AR (2014) Diagnostic accuracy of delirium diagnosis in pediatric intensive care: a systemic review. Crit Care 18:489.

81. Schieveld JN, van der Valk JA, Smeets I et al (2009) Diagnostic consideration regarding pediatric delirium: a review and a proposal for pediatric intensive care units. Intensive Care Med 35:1843-1849.

82. Alvarez RV, Palmer C, Czaja AS et al (2018) Delirium is a common and early finding in patients in the pediatric cardiac Intensive Care Unit. J Pediatr 195:206-212. 
83. Schieveld JN, Lousberg R, Berghmans E et al (2008) Pediatric illness severity measures predict delirium in a pediatric intensive care unit. Crit Care Med 36:1933-1936.

84. Schieveld JN, Strik JJ (2017) Pediatric Delirium: a worldwide PICU problem Crit Care Med 45:746747.

85. Traube C, Silver G, Gerber LM et al (2017) Delirium and mortality in critically ill children: epidemiology and outcomes of pediatric delirium. Crit Care Med 45:891-898.

86. Patel AK, Biagas KV, Clarke EC et al (2017) Delirium in children after cardiac bypass surgery. Pediatric Crit Care Med 18:165-171.

87. Ramirez CR, Gómez MLA, Vélez CAA et al (2019) Clinical characteristics, prevalence, and factors related to delirium in children of 5 to 14 years of age admitted to intensive care. Med Intensiva 43:147-155.

88. Traube C, Silver G, Reeder RW et al (2017) Delirium in Critical III Children: an international point prevalence study. Crit Care Med 45:584-590.

89. Traube C, Mauer EA, Gerber LM et al (2016) Cost associated with pediatric delirium in the ICU. Crit Care Med 44:e1175-e1179.

90. Smeets IA, Tan EY, Vossen HG et al (2010) Prolonged stay at the paediatric intensive care unit associated with paediatric delirium. Eur Child Adolesc Phychiatry 19:389-393.

91. Meyburg J, Ries M, Zirelonka M et al (2018) Cognitive and behavioural consequences of pediatric delirium: a pilot study. Pediatr Crit Care Med 19:e531-e537.

92. Silver G, Traube C, Gerber LM et al (2015) Pediatric delirium and associated risk factors: a singlecenter prospective observational study. Pediatr Crit Care Med 16:303-309.

93. Meyburg J, Dill ML, Traibe C et al (2017) Patterns of postoperative delirium in children. Pediatr Crit Care Med 18:128-133.

94. Turkel SB (2017) Pediatric delirium: recognition, management, and outcome. Curr Psychiatry Rep 19:101.

95. Malas N, Brahmbhatt K, McDermott C et al (2017) Pediatric delirium: evaluation, management, and special considerations. Curr Psychiatry Rep 19:65.

96. Simone S, Edwards S, Lardieri A et al (2017) Implementation of an ICU Bundle: an interprofessional quality improvement project to enhance delirium management and monitor delirium prevalence in a single PICU. Pediatr Crit Care Med 18:531-540.

97. Kawai Y, Weatherhead JR, Traube $C$ et al (2017) Quality improvement initiative to reduce Pediatric Intensive Care Unit noise pollution with the use of a pediatric delirium bundle. J Intensive Care Med 34:383-90.

98. Lee ES, Vidal C, Findling RL (2018) A focused review on the treatment of pediatric patients with atypical antipsychotics. J Child Adolesc Psychopharmacol 28:582-605.

99. Slooff VD, van den Dungen DK, van Beusekom BS et al (2018) Monitoring haloperidol plasma concentration and associated adverse events in critically ill children with delirium: first results of a 
clinical protocol aimed to monitor efficacy and safety. Pediatr Crit Care Med 19:e112-e119.

100. Sassano-Higgins S, Freudenberg N, Jacobson J et al (2013) Olanzapine reduces delirium symptoms in the critically ill pediatric patient. Journal of Pediatric Intensive Care 2:49-54.

101. Turkel SB, Jacobson JR, Tavaré CJ (2013) The diagnosis and management of delirium in infancy. J Child Adolesc Psychopharmacol 23:352-356.

102. Joyce C, Witcher R, Herrup E et al (2015) Evaluation of the safety of quetiapine in treating delirium in critically ill children: a retrospective review. J Child Adolesc Psychopharmacol 25:666-670.

103. Hilliard N, Brown S, Mitchinson S (2015) A case report of dexmedetomidine used to treat intractable pain and delirium in a tertiary palliative care unit. Palliat Med 29:278-281.

104. Kudchadkar SR Yaster M, Punjabi NM (2014) Sedation, sleep promotion, and delirium screening practices in the care of mechanical ventilated children: a wake-up call for the pediatric critical care community. Crit Care Med 42:1592-1600.

105. Smith HA, Boyd J, Fuchs DC et al (2011) Diagnosing delirium in critically ill children: validity and reliability of the pediatric confusion assessment method for the intensive care unit. Crit Care Med 39:150-157.

106. Traube C, Silver G, Kearney J et al (2014) Cornell Assessment of Pediatric Delirium: a valid, rapid, observational tool for screening delirium in the PICU. Crit Care Med 42:656-663.

107. Silver G, Kearney J, Traube C et al (2015) Delirium screening anchored in child development: the Cornell Assessment for Pediatric Delirium. Palliat Support Care 13:1005-1011.

108. Smith HA, Gangopadhyay M, Goben CM et al (2016) The Preschool Confusion Assessment Method for the ICU: valid and reliable monitoring for critically ill infants and children. Crit Care Med 44:592600.

109. Ista E, Te Beest H, van Rosmalen J et al (2018) Sophia Observation withdrawal Symptoms-Paediatric Delirium scale: a tool for early screening of delirium in the PICU Aust Crit Care 31:266-273.

110. Madden K, Burns MM, Tasker RC (2017) Differentiating delirium from sedative/hypnotic- related iatrogenic withdrawal syndrome lack of specificity in pediatric critical care assessment tools. Pediatr Crit Care Med 18:580-588.

111. Amigoni A, Vettore E, Brugnolaro V et al (2014) High doses of benzodiazepine predict analgesic and sedative drug withdrawal syndrome in paediatric intensive care patients. Acta Paediatr 103:e538e543.

112. da Silva PS, Rais ME, Fonseca TSM et al (2016) Opioid and Benzodiazepine Withdrawal Syndrome in PICU patients: which risk factors matter? J Addict Med 10:110-116.

113. Amigoni A, Mondardini MC, Vittadello I et al (2017) Withdrawal Assessment Tool-1 monitoring in PICU: a multicenter study on iatrogenic withdrawal syndrome. Pediatr Crit Care Med 18:e86-e91.

114. Anand KJ, Wilson DF, Berger J et al (2010) Tolerance and withdrawal from prolonged opioid use in critically ill children. Pediatrics 125:e1208-1225. 
115. Haenecour AS, Seto W, Urbain CM et al (2017) Prolonged dexmedetomidine infusion and drug withdrawal in critically ill children. J Pediatr Pharmacol Ther 22:453-460.

116. Best KM, Wypij D, Asaro LA et al (2017) Patient, process, and system predictors of iatrogenic withdrawal syndrome in critically ill children. Crit Care Med 45: e7-e15.

117. Best KM, Boullata JI, Curley MAQ (2015) Risk factors associated with iatrogenic opioid and benzodiazepine withdrawal in critically ill pediatric patients: A systematic review and conceptual model. Pediatr Crit Care Med 16:175-183.

118. Duceppe MA, Perreault MM, Frenette AJ et al (2019) Frequency, risk factors and symptomatology of iatrogenic withdrawal from opioids and benzodiazepine in critically ill neonates, children and adults: a systematic review of clinical studies. J Clin Pharm Ther 44:148-56.

119. Ista $E$, van Dijk M, Gamel $C$ et al (2008) Withdrawal symptoms in critically ill children after long-term administration of sedatives and/or analgesics: a first evaluation. Crit Care Med 36:2427-2432.

120. Best KM, Asaro LA, Franck LS et al (2016) Patterns of sedation weaning in critically III children recovering from acute respiratory failure. Pediatr Crit Care Med 17:19-29.

121. Gupta P, Whiteside W, Sabati A et al (2012) Safety and efficacy of prolonged dexmedetomidine use in critically ill children with heart disease. Pediatr Crit Care Med 13:660-666.

122. Czaja AS, Zimmermann JJ (2009) The use of dexmedetomidine in critically ill children. Pediatr Crit Care Med 10:381-386.

123. Johnson PN, Boyles KA, Miller JL (2012) Selection of the initial methadone regimen for the management of iatrogenic opioid abstinence syndrome in critically ill children. Pharmacotherapy 32:148-57.

124. Dervan LA , Yaghmai B, Watson RS et al (2017) The use of methadone to facilitate opioid weaning in pediatric critical care patients: a systematic review of the literature and meta-analysis. Paediatr Anaesth 27:228-239.

125. Honey BL, Benefield RJ, Miller JL et al (2009) Alpha2-receptor agonists for treatment and prevention of iatrogenic opioid abstinence syndrome in critically ill patients. Ann Pharmacother 43:1506-1511.

126. Capino AC, Miller JL, Johnson PN (2016) Clonidine for sedation and analgesia and withdrawal in critically ill infants and children. Pharmacotherapy 36:1290-1299.

127. Franck LS, Harris SK, Soetenga DJ et al (2008) The Withdrawal Assessment Tool-1 (WAT-1): an assessment instrument for monitoring opioid and benzodiazepine withdrawal symptoms in pediatric patients. Pediatr Crit Care Med 9:573-580.

128. Franck LS, Scoppettuolo LA, Wypij D et al (2012) Validity and generalizability of the Withdrawal Assessment Tool-1 (WAT-1) for monitoring iatrogenic withdrawal syndrome in pediatric patients Pain 153:142-148

129. Ista E, de Hoog M, Tibboel D et al (2013) Psychometric evaluation of the Sophia Observation withdrawal Symptoms -scale in critically ill children. Pediatr Crit Care Med 14:761-769. 
130. Anghelescu DL, Hamilton H, Faughnan LG et al (2012) Pediatric palliative sedation therapy with propofol: recommendations based on experience in children with terminal cancer. J Palliat Med 15:1082-1090.

131. Cherny NI, Radbruch L Board of the European Association for Palliative Care (2009) European Association for Palliative Care (EAPC) recommended framework for the use of sedation in palliative care. Palliat Med 23:581-593.

132. Wolfe J, Orellana L, Ullrich C et al (2015) Symptoms and distress in children with advanced cancer: Prospective patient- reported outcomes from the PediQUEST study. J Clin Oncol 33:1928-1935.

133. Twycross R (2019) Reflections on palliative sedation. Palliat Care 12:1178224218823511.

134. Johnson LM, Snaman JM, Cupit MC et al (2014) End-of-life care for hospitalized children. Pediatr Clin North Am 61:835-854.

135. Boss R, Nelson J, Weissman D et al (2014) Integrating Palliative Care Into the PICU: a report from the improving palliative care in the ICU advisory board. Pediatr Crit Care Med 15:762-767.

136. Anghelescu DL, Knapp E, Johnson LM et al (2017) The role of the pediatric anesthesiologist in relieving suffering at the end of life: when is palliative sedation appropriate in pediatrics? Pediatr Anesth 27:443-444.

137. Ramnarayan P, Craig F, Petros A et al (2007) Characteristics of deaths occurring in hospitalised children: changing trends. J Med Ethics 33:255-260.

138. Giannini A, Messeri A, Aprile A et al (2008) End-of-life decisions in pediatric intensive care. Recommendations of the Italian Society of Neonatal and Pediatric Anesthesia and Intensive Care (SARNePI). Paediatr Anaesth 18:1089-1095.

139. Italian Law March 15th 2010, n. 38. Disposizioni per garantire l'accesso alle cure palliative e alla terapia del dolore. GU n. 65, March 19th 2010.

140. Italian Law December 22nd 2017 n.219. Norme in materia di Consenso Informato e di Disposizioni Anticipate di Trattamento. GU n. 12, January 16th 2018.

141. Suttle ML L, Jenkins TL, Tamburro RF (2017) End-of-Life and bereavement care in Pediatric Intensive Care Units. Pediatr Clin North Am 64:1167-83.

142. Vallero SG, Lijoi S, Bertin D et al (2014) End-of-life care in pediatric neuro-oncology. Pediatr Blood Cancer 61:2004-2011.

143. Kiman R, Wuiloud AC, Requena ML (2011) End of life care sedation for children. Curr Opin Support Palliat Care 5:285-290.

144. Aindow A, Brook L. Essential medicines list for children (EMLC); Palliative Care. World Health Organization, June 2008 https://www.who.int/selection_medicines/committees/subcommittee/2/palliative.pdf?ua=1

145. Hooke MC, Grund E, Quammen H et al (2007) Propofol use in pediatric patients with severe cancer pain at the end of life. J Pediatr Oncol Nurs 24:29-34. 
146. Burns J, Jackson K, Sheehy K et al (2017) The use of dexmedetomidine in pediatric palliative care: a preliminary study. J Palliat Med 20:779-783.

147. O'Hara C, Tamburro RF, Ceneviva GD (2015) Dexmedetomidine for sedation during withdrawal of support. Palliat Care 9:15-18.

148. Miele E, Mastronuzzi A, Cefalo MG et al (2019) Propofol-based palliative sedation in terminally ill children with solid tumors: a case series. Medicine (Baltimore) 98:e15615.

149. Conway M, White N, St Jean C et al (2009) Use of continuous intravenous ketamine for end-stage cancer pain in children. J Pediatr Oncol Nurs 26:100-106.

150. Campbell-Fleming JM, Williams A (2008) The use of ketamine as adjuvant therapy to control severe pain. Clin J Oncol Nurs 12:102-107.

151. Taylor M, Jakacki R, May C et al (2015) Ketamine PCA for treatment of end-of-life neuropathic pain in pediatrics. Am J Hosp Palliat Care 32:841-848.

152. Breau LM, Burkitt C. (2009) Assessing pain in children with intellectual disabilities. Pain Res Manag 14:116-120.

153. Valkenburg AJ, de Leeuw TG, van Dijk M et al (2015) Pain in Intellectually Disabled Children: Towards Evidence-Based Pharmacotherapy? Pediatr Drugs 17:339-348.

154. Best KM, Asaro LA, Curley MAQ (2019) Sedation management for critically ill children with preexisting cognitive impairment. J Pediatr 206:204-11.e1.

155. Malviya S, Voepel-Lewis T, Burke C et al (2006) The revised FLACC observational pain tool: improved reliability and validity for pain assessment in children with cognitive impairment Pediatr Anaesth $16: 258-265$

156. Breau LM, Finley GA, Mc Grath PJ et al (2002) Validation of the Non-communicating Children's Pain Checklist-Postoperative version Anesthesiology 96:528-535

157. Di Bari A, Destrebecq A, Osnaghi F et al (2013) Traduzione e validazione in italiano della scala Revised FLACC per la valutazione del dolore nel bambino con grave ritardo mentale Pain Nursing Magazine 1:24-28.

158. Murgia M, Izzo R, Bettinelli A et al (2019) Validity and reliability of Italian version of the NonCommunicating Children's Pain Checklist: revised version. Eur J Phys Rehabil Med 55:89-94

159. Valkenburg AJ, Boerlage AA, Ista E et al (2011) The COMFORT-Behavior scale is useful to assess pain and distress in 0- to 3-year-old children with Down syndrome. Pain 152:2059-2064.

160. Valkenburg AJ, de Leeuw TG, Tibboel D et al (2009) Lower bispectral index values in children who are intellectually disabled. Anesth Analg 109:1428-1433.

161. Valkenburg AJ, van Dijk M, de Klein A et (2010) Pain management in intellectually disabled children: assesment, treatment, and translational research Dev Disabil Res Rev 16: 248-257.

162. Tomasi PA, Egger GF, Pallidis C et al (2017) Enabling development of paediatric medicines in Europe: 10 years of the EU Paediatric Regulation. Paediatr Drugs 19:505-513. 
163. Salvo I, Landoni G, Mucchetti M et al (2014) Use and reimbursement of off-label drugs in pediatric anesthesia: the Italian experience. Paediatr Anaesth 24:625-631.

164. Silver G, Traube C (2019) A systematic approach to family engagement: feasibility pilot of a pediatric delirium management and prevention toolkit. Palliative Support Care 17:42-45.

165. Craske J, Carter B, Jarman I et al (2019) Parent's experiences of their child's withdrawal syndrome: a driver for reciprocal nurse-parent partnership in withdrawal assessment. Intensive Crit Care Nurs 50:71-78.

\section{Tables}

TABLE 1: Research recommendations 


\begin{tabular}{|c|c|c|}
\hline $\begin{array}{l}\text { Recommendation } \\
1\end{array}$ & $\mathrm{R} 1$ & Studies on weaning from a prolonged infusion of dexmedetomidine \\
\hline $\begin{array}{l}\text { Recommendation } \\
2\end{array}$ & $\mathrm{R} 2$ & $\begin{array}{l}\text { Studies on the efficacy of drug rotation (analgesics or sedatives) } \\
\text { protocols }\end{array}$ \\
\hline \multirow{2}{*}{$\begin{array}{l}\text { Recommendation } \\
4\end{array}$} & R4.1 & Studies on the prolonged infusion of ketamine. \\
\hline & $\mathrm{R} 4.2$ & $\begin{array}{l}\text { Studies on efficacy and safety of other drugs used in difficult } \\
\text { analgesia and sedation (i.e sevoflurane) }\end{array}$ \\
\hline $\begin{array}{l}\text { Recommendation } \\
5\end{array}$ & R5 & Studies on length of treatment with neuromuscular blocking agents \\
\hline $\begin{array}{l}\text { Recommendation } \\
6\end{array}$ & R6 & Studies on continuous processed EEG in infants \\
\hline \multirow[t]{2}{*}{$\begin{array}{l}\text { Recommendation } \\
7\end{array}$} & $\mathrm{R} 7.1$ & $\begin{array}{l}\text { Studies on preventing and treatment of sleep disorders in Pediatric } \\
\text { Intensive Care Unit }\end{array}$ \\
\hline & R7.2 & $\begin{array}{l}\text { Studies on monitoring the quality of sleep in Pediatric Intensive Care } \\
\text { Unit with tools other than polysomnography }\end{array}$ \\
\hline \multirow[t]{2}{*}{$\begin{array}{l}\text { Recommendation } \\
8\end{array}$} & R8.1 & $\begin{array}{l}\text { Studies on the efficacy of alpha agonists in reducing the incidence of } \\
\text { delirium in critically ill patients }\end{array}$ \\
\hline & R8.2 & $\begin{array}{l}\text { Studies on the impact of reduction of modifiable factors in pediatric } \\
\text { delirium }\end{array}$ \\
\hline $\begin{array}{l}\text { Recommendation } \\
9\end{array}$ & R9 & $\begin{array}{l}\text { Studies on pharmacologic prevention and treatment of pediatric } \\
\text { delirium }\end{array}$ \\
\hline $\begin{array}{l}\text { Recommendation } \\
11\end{array}$ & $\mathrm{R} 11$ & Studies on weaning after ketamine and alpha agonists infusions \\
\hline $\begin{array}{l}\text { Recommendation } \\
12\end{array}$ & $\mathrm{R} 12$ & $\begin{array}{l}\text { Studies on preventing withdrawal syndrome from adopting strategies } \\
\text { of weaning or pharmacologic strategies }\end{array}$ \\
\hline $\begin{array}{l}\text { Recommendation } \\
15\end{array}$ & R15 & $\begin{array}{l}\text { Studies describing characteristics of pediatric palliative sedation in } \\
\text { children in Intensive Care Unit }\end{array}$ \\
\hline \multirow[t]{2}{*}{$\begin{array}{l}\text { Recommendation } \\
16\end{array}$} & R16.1 & $\begin{array}{l}\text { Studies on pharmacological interactions between patient's chronic } \\
\text { therapy and analgesic and sedative treatment administered in Intensive } \\
\text { Care Unit }\end{array}$ \\
\hline & R16.2 & $\begin{array}{l}\text { Studies on the development of tools dedicated to children with } \\
\text { developmental delay admitted to Pediatric Intensive Care Unit }\end{array}$ \\
\hline
\end{tabular}

\section{Figures}




\section{Search on 8 topics performed by the panellists adopting the requested criteria \\ 243 papers : \\ selection of the most relevant ones considering the full text}

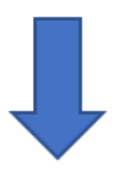

165 selected papers considered in the PICO development

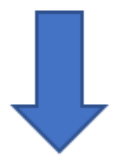

Quality analysis and evaluation of the risk of bias on the 9 Randomized Clinical Trials and the 51 Observational Studies

Figure 1

selection of literature 
PHYSICIAN

Every Day:
Minimizes physical/drug restraints
Removes unnecessary lines, tubes, devices
Contacts physiatrist/physiotherapist to perform physiotherapy
Uses a sleep protocol to minimize sleep interruption from 12 p.m. to 4 a.m.,
prescribing drugs to induce patient's sleep
If the Delirium scale is positive consider "the patient at risk of delirium"
bundle and give to caregivers the information related to pediatric delirium

For the patient at risk of delirium:
Compares today's delirium score to the previous day's one
Stops unnecessary treatment/drugs (benzodiazepine, antihistamines)
potentially triggering delirium
Treats withdrawal syndrome if the scale is positive
Promotes use of opiates/benzodiazepines sparing drugs
Starts antipsychotic therapy ideally contacting a psychiatrist

NURSE

Every day from 8 a.m. to 10 a.m.:
Lights patient's room
Presents him/herself to the patient and (if age is adequate) help him/her to
orientate (place, date, time)
Uses tools to communicate with the patient, even if he/her is non-verbal or
intubated
Promotes routine actions (physical therapy/videos/readings/laboratories),
also involving caregivers
Mobilizes patient
Brings patient's stuff and engages him/her with music, art, pet therapy
Identifies activities useful to involve caregivers in care of patient
Stimulates patient to weare his/her glasses/contact lenses/hearing aid

Every day from 8 p.m. to 11 p.m.:
Evaluates off lights
Presents him/herself to the patient and (if age is adequate) help him/her to
orientate (place, date, time)
Uses tools to communicate with the patient, even if he/her is non-verbal or intubated
Tries to apply patient's sleep routine
Evaluate with the team the opportunity to remove lines, tubes, devices
Switches off television, radio, devices
Mutes (using vibration tone) personal patient's mobile phone
Regulates room temperature
If delirium scale and withdrawal syndrome scale scores are high treats withdrawal
symptoms, if withdrawal syndrome scale score is low considers the use of
benzodiazepine sparing agents
Offers earplugs, earphones, headphones or neonatal earmuffs
Offers sleep mask
Offers thermal blanket

\section{Figure 2}

\section{bundle of pediatric delirium}




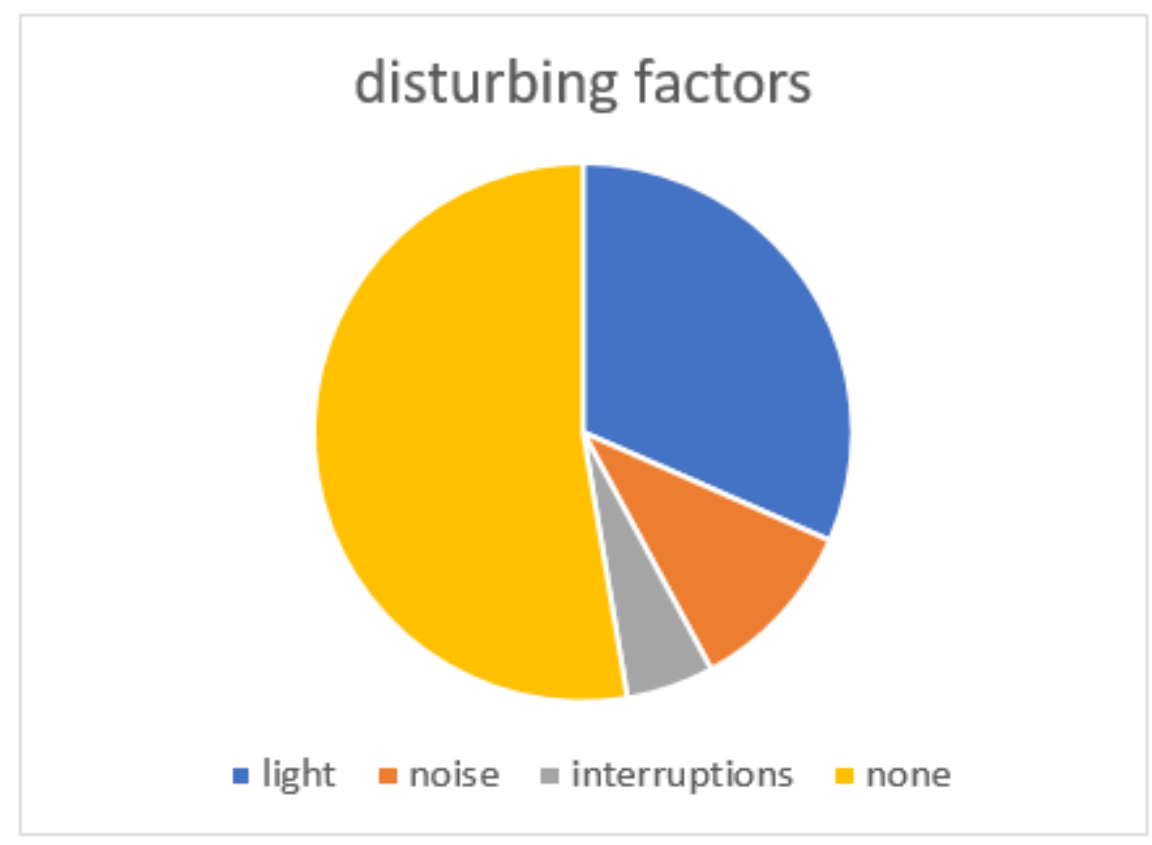

Figure 3

parents' opinions part A

\section{communication withdrawal syndrome and delirium}

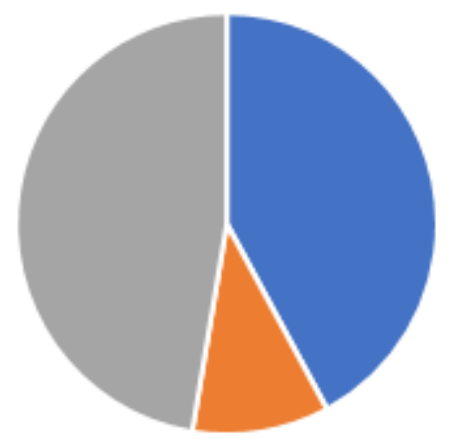

- complete $\quad$ only withdrawal syndrome a absent

\section{Figure 4}

parents' opinions part B 


\section{Supplementary Files}

This is a list of supplementary files associated with this preprint. Click to download.

- EJPSuppIMat1riskbias.xIsx

- EJPSuppIMat2aAnalgesiaandSedation.docx

- EJPSuppIMat2bDifficultAS.docx

- EJPSuppIMat2cNMBA.docx

- EJPSuppIMat2dSleep.docx

- EJPSuppIMat2eDelirium.docx

- EJPSupplMat2fWithdrawalSyndrome.docx

- EJPSuppIMat2gPalliativeSedation.docx

- EJPSuppIMat2hNeurodevelpmentalDelay.docx

- EJPSuppIMat3Tablesofrecommendations.docx

- EJPsupplmat4questionnaire.docx

- EJPSupplMat5drugs.docx

- EJPSuppIMath6druginteractions.docx 